\title{
RELAÇÃO DA CONCENTRAÇÃO DE PROGESTERONA COM O NÚMERO DE FOLÍCULOS E DIÂMETRO DO FOLÍCULO DOMINANTE EM OVELHAS SUBMETIDAS A JEJUM DURANTE A SINCRONIZAÇÃO DE CIOS
}

\author{
RINCÓN, Joao Alveiro Alvarado; \\ KIVEL, Taís Helena'; \\ PFEIFER, Luiz Francisco Machado ${ }^{3}$; \\ HAX, Lucas Teixeira ${ }^{4}$; \\ ANTUNES, Marcelo Moreira ; \\ DEL PINO, Francisco Augusto Burkert ${ }^{6}$; \\ CORRÊA, Marcio Nunes ${ }^{7}$; \\ SCHNEIDER, Augusto ${ }^{8}$.
}

\begin{abstract}
${ }^{1}$ Mestrando em Veterinária - UFPEL, ${ }^{2}$ Graduanda em Zootecnia - UFPEL, ${ }^{3}$ Pesquisador da EMBRAPA - Rondônia, ${ }^{4}$ Doutorando em Biotecnologia - UFPEL, ${ }^{5}$ Mestrando em Biotecnologia - UFPEL, ${ }^{6}$ Professor do Departamento de Bioquímica do Instituto de Química e Geociências - UFPEL, ${ }^{7}$ Professor Adjunto de Clínica Médica de Ruminantes - UFPEL, ${ }^{8}$ Professor Adjunto da Faculdade de Nutrição - UFPEL.
\end{abstract}

\section{RESUMO}

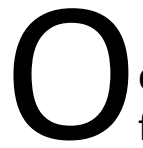
objetivo deste trabalho foi relacionar o efeito do jejum durante a sincronização de cios com o diâmetro do folículo ovulatório e contagem de folículos antrais. Para isto foi medida a concentração sanguínea de progesterona $\left(\mathrm{P}_{4}\right)$ no momento da remoção do dispositivo intravaginal liberador de progesterona (CIDR), assim como o número de folículos antrais e o diâmetro do folículo dominante de dez $(n=10)$ ovelhas mestiças submetidas ao jejum $(n=5)$ e alimentadas à vontade $(n=5)$, durante 84 horas, iniciando 48 horas antes da remoção do CIDR. A contagem dos folículos e a medição do folículo dominante foram determinadas após o abate das ovelhas e coleta individual dos ovários. Não houve diferença $(P>0,05)$ nas concentrações médias de $P_{4}$ no momento da inserção do CIDR entre grupos, porém no momento da remoção do CIDR as concentrações foram diferentes no grupo jejum $(4,07 \pm 0,32 \mathrm{ng} / \mathrm{mL})$ quando comparadas com o controle $(9,36 \pm 1,84$ $\mathrm{ng} / \mathrm{mL} ; \mathrm{P}<0,05)$. O diâmetro do folículo dominante e a contagem dos folículos antrais após a remoção do CIDR também não foram diferentes entre grupos $(P>0,05)$. Nas condições deste experimento o jejum aumentou a concentração sanguínea de $\mathrm{P}_{4}$ em ovelhas sincronizadas com CIDR, porém não teve efeito no número de folículos antrais, nem no tamanho do folículo dominante.

Palavras-chave: Ovino. Progestágeno. Restrição alimentar. CIDR. 


\section{INTRODUÇÃO}

A sincronização de estro em ovelhas é um avanço tecnológico que permite melhorar o desempenho reprodutivo e produtivo. Esta biotécnica permite concentrar a rotina de inseminação artificial e as parições, mesmo durante os períodos de anestro estacional (URIBE-VELÁSQUEZ et al., 2009). Um dos métodos mais usados é a utilização de esponjas intravaginais impregnadas com progestágenos, tais como acetato de medroxiprogesterona (MAP), acetato de fluorogestona (FGA) ou acetato de melengestrol (MGA), consorciados ou não com prostaglandina $F_{2} \alpha\left(P_{G} F_{2} \alpha\right)$ e/ou gonadotrofina coriônica equina (eCG) (DIAS et al., 2001). Outro dos métodos usados no controle do estro e ovulação em ovelhas é o dispositivo intravaginal liberador de progesterona (CIDR), que pode ser associado com eCG ou $\mathrm{PGF}_{2} \alpha$ (DIAS et al., 2001). Estudos mencionam $100 \%$ de presença de estro em animais que utilizaram CIDR contendo 0,33 g de progesterona $\left(\mathrm{P}_{4}\right)$ (NOGUEIRA et al., 2008; SOUSA et al., 2007). Quando é utilizada $P_{4}$ exógena ou seus análogos (MAP, FGA, MGA), ocorre regressão do corpo lúteo, mas o estro e, consequentemente, a ovulação serão manifestados quando a fonte de $\mathrm{P}_{4}$ for removida (CASTONGUAY et al., 1990).

O desempenho reprodutivo pode ser negativamente afetado em períodos de privação de alimento, com comprometimento do crescimento folicular no ovário (BUTLER et al., 2003). Em ovelhas, o efeito da restrição alimentar aguda em curto prazo não está totalmente definido, segundo Tatman et al. (1991) vacas e ovelhas adultas parecem ser resistentes a essa restrição, provavelmente devido à utilização dos nutrientes em fermentação presentes no rúmen, que pode variar de acordo com o grau de restrição alimentar e o estado reprodutivo dos animais (MACKEY et al., 2000). No entanto, existe uma relação inversa entre a ingestão de matéria seca e o nível sanguíneo de $\mathrm{P}_{4}$, desta maneira maior ingestão de matéria seca aumenta o fluxo sanguíneo para a veia porta hepática, levando assim mais sangue ao fígado, ocorrendo a maior catabolização da $\mathrm{P}_{4}$ e consequente redução dos seus níveis séricos (SANGSRITAVONG et al., 2002).

Na dinâmica folicular, após o recrutamento, vários folículos pequenos vão aumentando seu diâmetro e suas funções endócrinas, sendo que posteriormente somente um se tornará o dominante até a ovulação ou atresia com um diâmetro de 4-12 mm (DUGGAVATHI et al., 
2003). Nas ovelhas, folículos com diâmetros maiores que 2-3 mm são totalmente dependentes de gonadotrofinas (SCARAMUZZI et al., 1993) e seu crescimento nesta fase é muito rápido; aproximadamente $1 \mathrm{~mm} /$ dia (LEYVA et al., 1998). Após a seleção do folículo dominante, ele vai impedir o desenvolvimento dos outros folículos da mesma onda, diminuindo a secreção de FSH e aumentando a de LH mediante um feedback positivo entre o folículo dominante e o eixo hipotálamo-hipófise que vai culminar na ovulação (DRIANCOURT, 2001). Os níveis de $\mathrm{P}_{4}$ exercem influência na dinâmica folicular, evidenciando que ondas desenvolvidas sob elevada e contínua concentração deste hormônio apresentam folículos dominantes com tamanho menor que aqueles pertencentes a ondas desenvolvidas sob baixos níveis de $\mathrm{P}_{4}$ (GINTHER; KOT, 1994). Altas concentrações de $\mathrm{P}_{4}$ podem inibir o crescimento do folículo dominante (URIBE-VELÁSQUEZ et al., 2008b), visto que a $\mathrm{P}_{4}$ exerce um feedback negativo no eixo hipotálamo-hipófise decrescendo a frequência dos pulsos de LH (LEYVA et al., 1998).

Baseado nestas considerações, o objetivo deste trabalho foi relacionar o efeito do jejum sobre a concentração sanguínea de $\mathrm{P}_{4}$, diâmetro do folículo dominante e número de folículos antrais em ovelhas submetidas a um protocolo de sincronização a base de CIDR.

\section{MATERIAL E MÉTODOS}

O comitê de ética em experimentação animal da Universidade Federal de Pelotas aprovou todos os procedimentos realizados neste experimento, sob o protocolo 8593.

Foram utilizadas ovelhas multíparas $(n=10)$ mestiças (Corriedale X Texel), não lactantes, 2,5

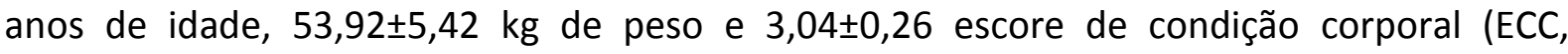
1=magra e 5=obesa) (RUSSEL, 1991) durante a estação de monta no sul do Brasil $\left(31^{\circ} 48^{\prime}\right.$ S, $\left.52^{\circ} 24^{\prime} \mathrm{W}\right)$. As ovelhas foram alojadas em baias cobertas $(2,0 \times 3,5 \mathrm{~m})$ e agrupadas por ECC e peso em dois grupos. A dieta foi baseada em feno de tifton (1,5 $\mathrm{Kg}$ de matéria seca) e concentrado (0,5Kg de MS; 47,5 g de extrato etéreo, 193,4 g de proteína bruta, 1194,5 g de fibra em detergente neutro e 931,5 g de fibra em detergente ácido) de acordo com o NRC (1985), e aceso à água à vontade. 
As ovelhas foram submetidas a uma pré-sincronização com esponja intravaginal contendo acetato de medroxiprogesterona (60 mg; DEG, São Paulo, Brasil) durante 14 dias. A detecção do estro foi realizada após a remoção da esponja por observação visual três vezes ao dia; às $08 \mathrm{~h}, 12 \mathrm{~h}$ e $19 \mathrm{~h}$, em que ovelhas detectadas em estro foram consideradas no dia 0 do próximo ciclo estral. Aproximadamente 11 dias após a detecção do estro, todos os animais receberam uma injeção de $125 \mu \mathrm{g}$ de cloprostenol sódico (IM, Ciosin $\AA$, Schering-Coopers, Cotia, Brasil) e $36 \mathrm{~h}$ depois, $100 \mu \mathrm{g}$ de gonadolerina (IM, Fertigen®, Tortuga, Sto. Amaro, Brasil) a fim de induzir a ovulação dentro de 24 h. Vinte quatro horas após a injeção de gonadolerina (tempo esperado de ovulação e emergência da nova onda folicular) foi implantado o CIDR (CIDR®, InterAg, Hamilton, Nova Zelândia) que permaneceu nas ovelhas durante 6 dias, quando foi injetado $125 \mu \mathrm{g}$ de cloprostenol sódico. Dois dias antes da remoção do CIDR os animais foram estabelecidos como grupo controle; que recebeu uma dieta de manutenção durante todo o período experimental $(n=5)$ e grupo jejum; no qual foi removida completamente a alimentação até o fim do experimento com apenas acesso à água $(n=5)$.

As amostras de sangue foram coletadas no momento da inserção do CIDR e no momento da remoção, mediante punção venosa jugular em tubos vacutainer (BD Diagnostics, São Paulo, Brasil) contendo EDTA. Os tubos foram centrifugados (1000 rpm por 20 minutos), separado o plasma e armazenado em micro tubos a $-80{ }^{\circ} \mathrm{C}$ até a avaliação. As concentrações de $\mathrm{P}_{4}$ foram avaliadas mediante radioimunoensaio com kit comercial (DSL-3400, Diagnostics Systems Laboratories Inc., Webster, TX, USA).

O jejum começou $48 \mathrm{~h}$ antes da implantação do CIDR e terminou $36 \mathrm{~h}$ após sua remoção, quando todas as ovelhas foram abatidas em um abatedouro local e os ovários foram recolhidos em frascos individuais esterilizados contendo solução salina e transportados para o laboratório em gelo. Neste momento, foi dissecado e medido o diâmetro do maior folículo. Além disso, todos os folículos visíveis na superfície ovariana foram medidos e contados. Os folículos foram classificados em pequenos $(<2 \mathrm{~mm})$, médios $(2-4 \mathrm{~mm})$ e grandes $(>4 \mathrm{~mm})$ conforme o descrito por Sarath et al. (2008). 
A análise estatística foi realizada usando o software Graphpad Prism 5 (GraphPad Software Inc., La Jolla, CA, USA). Para analisar a concentração de $\mathrm{P}_{4}$ e diâmetro folicular foi usado o teste de "t" e para a contagem de folículos foi usado o teste de Mann-Whitney.

\section{RESULTADOS E DISCUSSÃO}

No momento da inserção do CIDR não foram observadas diferenças nas concentrações plasmáticas de $\mathrm{P}_{4}$ entre tratamentos (tabela 1 ), isto provavelmente pela ausência de um corpo lúteo funcional, como consequência da aplicação exógena de $\mathrm{PGF}_{2} \alpha$ dois dias antes da inserção do CIDR. Já no momento da remoção do CIDR, o grupo jejum $(9,35 \pm 1,84 \mathrm{ng} / \mathrm{ml})$ apresentou maior concentração média de $P_{4}$ que o grupo controle $(4,07 \pm 0,32 \mathrm{ng} / \mathrm{mL}$; $P=0,02$ ), semelhante ao descrito em outros estudos (ALEXANDER et al., 2007; KIYMA et al., 2004) em que ovelhas ovariectomizadas tratadas com CIDR evidenciaram concentrações de $\mathrm{P}_{4}$ aproximadamente duas vezes maiores no grupo jejum do que o grupo com alimentação à vontade.

Tabela 1 - Concentrações plasmáticas médias de $P_{4}$ de ovelhas submetidas ou não a jejum.

\begin{tabular}{cccc}
\hline Momento & Controle $(\mathbf{n g} / \mathbf{m L})$ & Jejum $(\mathbf{n g} / \mathbf{m L})$ & Valor de $\mathbf{P}$ \\
\hline Inserção do CIDR & $0,86 \pm 0,15$ & $0,9 \pm 0,10$ & $>0,05$ \\
Remoção do CIDR & $4,07 \pm 0,32$ & $9,35 \pm 1,84$ & 0,02 \\
\hline
\end{tabular}

\pm erro padrão

Tanto nos grupos controle e jejum no momento da inserção do CIDR, quanto no grupo controle no momento da remoção do CIDR, as concentrações de $\mathrm{P}_{4}$ foram semelhantes ao descrito em estudos prévios (COELHO et al., 2000; MINTON et al., 1990; SASA et al., 2002; URIBE-VELÁSQUEZ et al., 2008a).

Os achados de Kiyma et al. (2004) descartam efeito do ovário no aumento das concentrações de $\mathrm{P}_{4}$, baseado nisto, sugere-se que o efeito do jejum no aumento das concentrações de $\mathrm{P}_{4}$ em ovelhas está relacionado com a diminuição de fluxo na veia porta hepática, ocasionado pela redução na ingesta de alimento. Desta maneira, uma menor quantidade de sangue passa pelo fígado, que é o local onde ocorre a maior catabolização da 
$\mathrm{P}_{4}$ (SANGSRITAVONG et al., 2002), catabolizando menos $\mathrm{P}_{4}$ e elevando a concentração sanguínea deste hormônio.

Neste trabalho não foi observado efeito do jejum sobre o diâmetro do folículo dominante $(P>0,05)$, apresentando diâmetro médio de $7,1 \pm 0,56 \mathrm{~mm}$ no grupo controle e $7,4 \pm 0,37 \mathrm{~mm}$ no grupo jejum, resultados que diferem dos reportados por Ginther e Kot (1994); UribeVelásquez, et al. (2008b) que mencionam que elevadas concentrações de $\mathrm{P}_{4}$ podem ter um efeito inibitório sobre o crescimento do folículo dominante, principalmente por exercer um feedback negativo no hipotálamo e hipófise, regulando a liberação das gonadotrofinas e, principalmente, na liberação de LH. No entanto, neste estudo apesar das concentrações de $\mathrm{P}_{4}$ terem sido aproximadamente duas vezes maiores no grupo jejum do que no controle não se observou alteração do crescimento do folículo dominante como indicado anteriormente. O diâmetro do folículo dominante neste estudo foi maior que o reportado por UribeVelásquez et al. (2009) e que em condições similares realizaram a medição do folículo dominante em ovelhas das raças Bergamácia e Santa Inês. As variações no diâmetro folicular podem estar relacionadas à resposta endócrina da raça e/ou ao protocolo de sincronização utilizado (DIAS et al., 2001; LENZ et al., 2008), além disso a sincronização pré-protocolo com esponja intravaginal e após a utilização do CIDR pode influenciar no aumento no diâmetro folicular (VIÑOLES et al., 2001).

A contagem de folículos antrais não apresentou diferença entre os grupos controle e jejum em nenhum dos tamanhos $(P>0,05)$ (tabela 2$)$, diferente do relatado por Uribe-Velásquez et al. (2008a) que mencionam que altas concentrações de $P_{4}$ têm influencia negativa sobre a dinâmica folicular, no entanto, apesar de apresentar diferença nas concentrações de $P_{4}$, não houve variação no número de folículos. O número de folículos pequenos neste estudo foi maior do que reportado por Uribe-Velásquez et al. (2008b), podendo ser atribuída à eficiência do método de contagem, já que neste estudo a contagem foi realizada diretamente na superfície dos ovários, entretanto o autor mencionado anteriormente utilizou ultrassom para realizar a contagem e isto pode dificultar a visualização dos folículos de menor tamanho. 
Tabela 2 - Médias da contagem de folículos antrais de ovelhas mantidas ou não em jejum.

\begin{tabular}{cccc}
\hline Tamanho & Controle & Jejum & Valor de $\mathbf{P}$ \\
\hline Pequeno $(<2)$ & $14,2 \pm 4,42$ & $9,0 \pm 2,58$ & 0,40 \\
Médio $(2-4)$ & $0,8 \pm 0,37$ & $1,2 \pm 0,37$ & 0,50 \\
Grande $(>4)$ & $1,2 \pm 0,20$ & $1,2 \pm 0,20$ & 0,88 \\
Contagem total & $16,2 \pm 4,63$ & $11,4 \pm 2,66$ & 0,46 \\
\hline
\end{tabular}

\pm erro padrão

O número de folículos de tamanho médio e grande foram similares no grupo controle e jejum, com valores de $0,8 \pm 0,37,1,2 \pm 0,20$ e 1,2 $\pm 0,37,1,2 \pm 0,20$, respectivamente, semelhante ao encontrado por Uribe-Velásquez et al. (2008b). Porém, a contagem de folículos totais foi maior que a descrita por Uribe-Velásquez et al. (2002), como consequência do maior número de folículos pequenos encontrados neste estudo.

\section{CONCLUSÃO}

$\mathrm{O}$ jejum aumenta a concentração sanguínea de $\mathrm{P}_{4}$ em ovelhas sincronizadas com CIDR, porém esse nível de $\mathrm{P}_{4}$ não teve efeito no número de folículos antrais, nem no tamanho do folículo dominante após a remoção do CIDR.

\section{AGRADECIMENTOS}

Este projeto foi financiado pela CAPES (Protocolo 106/2007), além disso, o primeiro autor recebe bolsa pela CAPES. 


\section{RELATIONSHIP OF PROGESTERONE CONCENTRATIONS WITH THE NUMBER OF FOLLICLES AND DOMINANT FOLLICLE DIAMETER IN SHEEP SUBMITTED TO FASTING DURING ESTRUS SYNCHRONIZATION}

\section{ABSTRACT}

$\mathrm{T}$ he aim of this study was to relate the effect of fasting with the ovulatory follicle diameter and antral follicle count in ewes during estrus synchronization. The concentrations of seric progesterone $\left(\mathrm{P}_{4}\right)$ at the time of removal of the progesterone releasing intravaginal device (CIDR) were measured, as well as the number of antral follicle and the diameter of the dominant follicle of ten $(n=10)$ crossbred ewes, submitted to fasting $(n=5)$ and fed ad libitum $(n=5)$ for 84 hours, starting 48 hours before CIDR removal. The counting of follicles and the measure of the dominant follicle were determined after slaughter and collection of individual ovaries. There was no difference $(P>0.05)$ in the average concentrations of $\mathrm{P}_{4}$ at the time of CIDR insertion between groups, but the time of CIDR removal concentrations of $\mathrm{P}_{4}$ were higher for fasting $(9.36 \pm 1.84 \mathrm{ng} / \mathrm{mL})$ than in the control group $(4.07 \pm 0.32 \mathrm{ng} / \mathrm{mL})(P<0.05)$. The diameter of the dominant follicle and the count of antral follicles were also not different $(P>0.05)$. In this experiment the conditions of fasting increased blood concentrations of $\mathrm{P}_{4}$ in ewes synchronized with $\mathrm{CIDR}$, but had no effect on the number of antral follicles or the size of the dominant follicle after CIDR removal.

Keywords: Sheep. Progestin. Feed Restriction. CIDR.

\section{RELACIÓN DE LA CONCENTRACIÓN DE PROGESTERONA CON EL NÚMERO DE FOLÍCULOS Y DIÁMETRO DEL FOLÍCULO DOMINANTE EN OVEJAS SOMETIDAS A AYUNO DURANTE LA SINCRONIZACIÓN DE CELOS}

\section{RESUMEN}

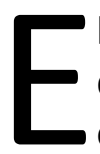

I objetivo de este trabajo fue relacionar el efecto del ayuno durante la sincronización de celos con el diámetro del folículo ovulatorio y el número de folículos antrales. Para esto, fue medida la concentración sanguínea de progesterona $\left(P_{4}\right)$ en el momento que fue retirado el dispositivo intravaginal liberador de progesterona (CIDR), así como el número de folículos antrales y el diámetro del folículo dominante de diez $(n=10)$ ovejas mestizas sometidas a ayuno $(n=5)$ y alimentadas ad libitum $(n=5)$, durante 48 horas antes de retirar el CIDR. Los folículos fueron contados y medidos después del sacrificio de las ovejas y también fue realizada colecta individual de los ovarios. No hubo diferencia $(P>0,05)$ en las concentraciones de $\mathrm{P}_{4}$ entre grupos en el momento en que fue colocado o CIDR, sin embargo 
en el momento que fue retirado las concentraciones fueron diferentes en el grupo con ayuno $(4,07 \pm 0,32 \mathrm{ng} / \mathrm{mL})$ en comparación al grupo control $(9,36 \pm 1,84 \mathrm{ng} / \mathrm{mL} ; \mathrm{P}<0,05)$. El diámetro del folículo dominante y el número de los folículos antrales después de la retirada del CIDR no fueron diferentes entre grupos $(P>0,05)$. En las condiciones de este estudio, el ayuno aumentó la concentración sanguínea de $\mathrm{P}_{4}$, sin embargo no tuvo efecto sobre el número de folículos antrales, ni en el tamaño del folículo dominante.

Palabras clave: Ovino. Progestágeno. Restricción Alimentar. CIDR.

\section{REFERÊNCIAS}

ALEXANDER, B. M.; KIYMA, Z.; MCFARLAND, M.; et al. Influence of short-term fasting during the luteal phase of the estrous cycle on ovarian follicular development during the ensuing proestrus of ewes. Animal Reproduction Science, Amsterdam, n. 97, p. 356-363, 2007.

BUTLER, S. T.; MARR, A. L.; PELTON, S. H.; et al. Insulin restores GH responsiveness during lactation-induced negative energy balance in dairy cattle: effects on expression of IGF-I and GH receptor 1A. Journal of Endocrinology, v. 176, p. 205-217, 2003.

CASTONGUAY, F.; DUFOUR, J. J.; MINVIELLE, F.; et al. Follicular dynamics and dominance in Boorola $x$ Finnish Landrace and Boorola $x$ Suffolk ewes heterozygous for the F gene. Journal of Reproduction and Fertility, v. 89, p. 193-203, 1990.

COELHO, L. A.; RODRIGUES, P. A.; SASA, A.; et al. Concentrações plasmáticas de progesterona em borregas lanadas e deslanadas durante a estação reprodutiva. In: REUNIÃO ANUAL DA SOCIEDADE BRASILEIRA DE ZOOTECNIA, 37, 2000, Viçosa. Anais. Viçosa:

Sociedade Brasileira de Zootecnia, 2000. CD-ROM.

DIAS, F. E. F.; LOPES, E. S.; VILLARROEL, A. B. S.; et al. Sincronização do estro, indução da ovulação e fertilidade de ovelhas deslanadas após tratamento hormonal com gonadotrofina coriônica equina. Arquivo Brasileiro de Medicina Veterinária e Zootecnia, v. 53, n. 5, p. 618623, 2001.

DRIANCOURT, M. A. Regulation of ovarian dynamics in farm animals. Implications for manipulation of reproduction. Theriogenology, v. 55, p. 1211-1239, 2001.

DUGGAVATHI, R.; BARTLEWSKI, P. M.; BARRETT, D. M. W.; RAWLINGS, N. C. Use of highresolution trasrectal ultrasonography to asses changes in numbers of small ovarian antral follicles and their relationships to the emrgence of follicular waves in cyclic ewes.

Theriogenology, v. 60, p. 495-510, 2003.

GINTHER, O. J.; KOT, K. Follicular dynamics during the ovulatory season in goats.

Theriogenology, v. 42, p. 987-1001, 1994. 
KIYMA, Z.; ALEXANDER, B. M.; VAN KIRK, E. A.; et al. Effects of reed restriction on reproductive and metabolic hormones in ewes. Journal Animal Science, Champaign, n. 82, p. 2548-2557, 2004.

LENZ SOUZA, M. I.; URIBE-VELÁSQUEZ, L. F.; OBA, E.; et al. Secreção de esteróides ovarianos, em ovelhas mestiças de raças exploradas para corte, em distintos momentos reprodutivos, no estado de são Paulo. Ciência Animal Brasileira, Goiânia, v. 9, n. 4, p. 1107-1113, 2008.

LEYVA, V.; BUCKRELL, B. C.; WALTON, J. S. Regulation of follicular and ovulation in ewes by exogenous progestagen. Theriogenology, v. 50, p. 395-416, 1998.

MACKEY D. R.; WYLIE A. R.; SREENAN J. M.; et al. The effect of acute nutritional change on follicle wave turnover, gonadotropin, and steroid concentration in beef heifers. Journal of Animal Science, n. 78, p. 429-442, 2000.

MINTON, J. E.; COPPINGER, T. R.; SPAETH, C. W.; et al. Poor reproductive response of anestrous Suffolk ewes to ram exposure is not due to failure to secrete luteinizing hormone acutely. Journal of Animal Science, Champaign, v. 69, p. 33114-3320, 1990.

NRC. Nutrient requirements of sheep. Washington: National Academic Press, 1985. 99p.

NOGUEIRA, D. M.; LOPES JÚNIOR, E. S.; CHRISTILIS, M.; et al. Fertilidade de cabras leiteiras exploradas na região semi-árida do Nordeste do Brasil após inseminação artificial e utilização por até três vezes do dispositivo de liberação controlada de drogas (CIDR) para sincronização do estro. REUNIÃO ANUAL DA SOCIEDADE BRASILEIRA DE ZOOTECNIA, 45, 2008, Lavras Anais. Lavras: UFLA/SBZ, 2008.

RUSSEL, A. Body condition scoring of sheep. In: BODEN, E. (Ed.). Sheep and goat practice. Oxford: Bailliére Tindall, 1991. p. 03-10.

SANGSRITAVONG, S.; COMBS, D. K.; SARTORI, R.; et al. High feed intake increases liver blood flow and metabolism of progesterone and estradiol-17ß in dairy cattle. Journal of Dairy Science, v. 85, p. 2831-2842, 2002.

SARATH, T.; MEHROTRA, S.; AGARWAL, S. K.; et al. Effect of insulin administration on ovarian function and estrus induction in acyclic goats. Animal Reproduction Science, Amsterdam, $\mathrm{n}$. 108, p. 216-225, 2008.

SASA, A.; TESTON, D. C.; RODRIGUEZ, P. A.; et al . Concentrações plasmáticas de progesterona em borregas lanadas e deslanadas no período de abril a novembro, no Estado de São Paulo. Revista Brasileira de Zootecnia, v. 31, n. 3, p. 1150-1156, 2002.

SCARAMUZZI, J.; ADAMS, N. R.; BAIRD, D. T.; et al. A model for follicle selection and the determination of ovulation rate in the ewe. Reproduction Fertility Development, v. 5, p. 459-478, 1993. 
SOUZA, J. M. G.; GOMES, L. M.; MONTEIRO, P. L. J.; et al. Uso de protocolos curtos para indução de estro em ovelhas Santa Inês. CONGRESSO BRASILEIRO DE REPRODUÇÃO ANIMAL, 17, 2007, Curitiba. Anais. Belo Horizonte: CBRA, 2007.

TATMAN, W. R.; JUDKINS, M. B.; KRYSL, L. J.; MOSS, G. E. Gastrointestinal digesta passage and fermentation patterns associated with restricted intake of a low quality forage in ewes. Small Ruminant Research, n. 4, p. 393-399, 1991.

URIBE-VELÁSQUEZ, L. F.; OBA, E.; LARA-HERRERA, L. C.; et al . Respostas endócrinas e ovarianas associadas com o folículo dominante da primeira onda folicular em ovelhas sincronizadas com CIDR ou PGF2 alfa. Revista Brasileira de Zootecnia, v. 31, n. 2, p. 944-953, 2002.

URIBE-VELÁSQUEZ, L. F.; LENZ, M. I.; OSORIO, J. H. Origem e características do folículo pré ovulatório depois de luteólise induzida em diferentes estágios da fase luteal do ciclo estral em ovelhas. Veterinária e Zootecnia, v. 2, n. 1, p. 32-41, 2008 a.

URIBE-VELÁSQUEZ, L. F.; OBA, E.; SOUZA, M. I. L. Efeitos da progesterona exógena sobre o desenvolvimento folicular em ovelhas. Arquivo Brasileiro de Medicina Veterinária e Zootecnia, v. 60, n. 1, p. 58-65, 2008b.

URIBE-VELÁSQUEZ, L. F.; RESTREPO CADAVID, R.; OSORIO, J. H. Respostas foliculares e endócrinas em ovelhas após sincronização do estro usando progesterona, prostaglandinas (PGF2 $\alpha$ ) e gonadotrofinas. Veterinária e Zootecnia, v. 3, n. 2, p. 14-27, 2009.

VIÑOLES, C.; FORSBERG, M.; BANCHERO, G.; RUBIANES, E. Effect of long-term and short-term progestagen treatment on follicular development and pregnancy rate in cyclic ewes.

Theriogenology, Amsterdam, n. 55, p. 993-1004, 2001. 